\title{
ACM Springer Mobile Networks and Applications (MONET) Journal Special Issue on Cooperatively Networked Femtocells
}

\author{
David Lopez-Perez • Xiaoli Chu • Guillaume de la Roche • \\ Athanasios V. Vasilakos • Mischa Dohler
}

Published online: 20 September 2012

(C) Springer Science+Business Media, LLC 2012

\section{Editorial:}

The proliferation of new applications, e.g., mobile TV, Internet gaming, large file transfer, and the development of user terminals, e.g., smart phones and notebooks, has dramatically increased user traffic and network load. Moreover, with the number of wireless subscribers expected to triple over the next 5 years, it is obvious that current networks will not be able to satisfy customer demands in the near future. In order to meet this traffic growth and provide service to their users, vendors and operators are working on the development of new technologies and cellular standards. Within them, heterogeneity in network deployment has been heralded as the most promising way of increasing both coverage and capacity of future wireless systems. Thus, it is expected that new elements such as remote radio heads, picocells, femtocells, and relay nodes will be deployed overlying macrocells. In this way, networks will

D. Lopez-Perez $(\square)$

Autonomous Networks and System Research Department,

Bell Labs, Alcatel-Lucent,

Dublin, Ireland

e-mail: david.lopez.work@gmail.com

X. Chu

Department of Electronic and Electrical Engineering,

University of Sheffield,

Sheffield, UK

G. de la Roche

Mindspeed Technologies,

Sophia Antipolis,

Nice, France

\author{
A. V. Vasilakos \\ Department of Computer and Telecommunications Engineering, \\ University of Western Macedonia, \\ Kozani, Greece \\ M. Dohler \\ CTTC, \\ Barcelona, Spain
}

be closer to users, and system capacity will be enhanced through a better spatial spectrum reuse.

However, although heterogeneous networks are envisioned to support the increasing data traffic demand and meet the requirements imposed for the fourth generation of mobile networks, they also lead to new technical challenges never faced before. For example, due to the larger number of cells and thus of cell boundaries, the management of interference becomes an intricate problem. Since centralized network planning and optimization cannot deal with the individualistic nature of user-deployed cells, e.g., femtocells, a key to the success of heterogeneous networks is the cooperation between nodes in a decentralized and distributed manner. Such cooperation is the only way to ensure a proper network operation. However, cooperation is not easy to achieve due to different issues and threats imposed by the network itself, e.g., limited back-haul capabilities, dynamics of traffic and radio channel, energy consumption, operational costs, etc.

This special issue features six selected papers with high quality:

The first article, "Managing Femto to Macro Interference without X2 Interface Support through POMDP", co-authored by Ana Galindo-Serrano and Lorenza Giupponi, presents a self-organized downlink power control for interference management in macrocellfemtocells co-channel deployments. The authors provide a completely autonomous framework, considering 3GPP LTE Release 11 and the non-availability of X2 interfaces between evolved NodeBs (eNBs) and HeNBs. In this situation, HeNBs make autonomous decisions without receiving any feedback from the macrocell network. The network is modeled as a multi-agent system where each node is an independent agent able to learn downlink power allocation policies for different interference situations through Reinforcement Learning techniques. In order to deal with the lack of information in the scenario, 
the authors rely on the theory of Partially Observable Markov Decision Process (POMDP), which works on the basis of a set of beliefs that HeNBs build considering the impact they have on the macrocell system. Moreover, in order to gather this system performance information, the authors propose that HeNBs use spatial interpolation techniques, such as ordinary Kriging. Results show that the proposed approach allows HeNBs to autonomously learn a good power allocation policy that allows them to coexist with the macrocell network without introducing extra signaling overhead.

The second article titled "McPAO: A Distributed Multi-Channel Power Allocation and Optimization Algorithm for Femtocells", from Yang Yang, Xiaojin Zheng, Jing Xu, Jiang Wang, Xiaoying Zheng, Yong Teng and Kari Horneman, formulates a multi-channel transmit power allocation problem that allows to maximize the overall system throughput under complex transmit power constraints. The authors apply Lagrangian duality techniques to make the problem decomposable and propose a distributed iterative sub-gradient algorithm, namely Multi-channel Power Allocation and Optimization (McPAO), to solve it. Numerical results show that McPAO can improve the overall system throughput by $18 \%$, comparing to fixed transmit power allocation schemes. In addition, the authors study the impact of errors in gradient direction estimation, which are caused by limited or delayed information exchange among femtocells in realistic situations. These errors will be propagated into the sub-gradient algorithm and, subsequently, affect the overall performance of McPAO. A rigorous analytical approach is developed to prove that McPAO can always achieve a bounded overall throughput performance very close to the global optimum.

The next article "Traffic and Mobility Management in Networks of Femtocells" investigates a new system architecture conceived for efficiently deploying femtocell networks, in which groups of femtocells in the same administrative domain cooperate towards a global performance improvement. The authors (Jaime Ferragut, Josep Mangues-Bafalluy, José Núñez-Martínez and Frank Zdarsky) propose femtocell modifications together with a new network entity called Local Femto Gateway (LFGW), which allows offloading a high volume of control and data traffic from the core network to functional entities in the femtocell network. The authors focus on building blocks related to traffic and mobility management where a two-level routing approach is discussed. The high-level routing is carried out by the mobile network layer, and solutions for efficient handover, local breakout, and local location management are presented. The low-level routing is carried out by the transport network layer, and distributed routing solution for a large-scale, all-wireless femtocell network is presented. Overall, these architectural improvements render the proposed architecture a promising approach for efficient traffic management in large-scale femtocell deployments, hence making them a scalable solution. The fourth article, "Energy Efficient Networking with IEEE 802.16 m Femtocell Low Duty Mode", coauthored by Hung-Yu Wei, Ching-Chun Kuan and GuanYu Lin, studies low duty mode femtocell operation in order to efficiently mitigate interference between macrocells and femtocells. The authors propose a new mechanism for integrating the low duty mode in femtocells and the sleep mode in user equipment to achieve a higher powersaving efficiency. Based on the IEEE $802.16 \mathrm{~m}$ system architecture, the authors build a practical system model and analyze the performance of the proposed mechanisms. Result shows that with simple modifications of the sleep-cycle operation, the proposed mechanisms can significantly reduce the power consumption of femtocells, leading to energy efficient operation of femtocells.

The fifth article titled "Energy Conservation via Antenna Scheduling in Fiber-Connected Femto Base Stations", from Haoming Li, Alireza Attar and Victor C. M. Leung, formulates the energy consumption of broadband wireless access with fiber connected massively distributed antennas (BWA-FMDA) architecture under a generalized framework. Moreover, the authors propose an optimization tool based on coordinated multipoint transmission (femto-CoMP) for femtocell clustered networks to maximize energy efficiency by adjusting the number of transmit antennas and controlling transmit power in zero-forcing beamforming. Based on the analytical results, the authors group every two neighboring antennas in multiple femto-CoMP configurations, and propose a new network configuration scheme that uses antenna scheduling to jointly improve spectral and energy efficiency. Compared with standalone femtocells, the proposed scheme is shown to increase energy efficiency in a typical office building by $64 \% \sim 160 \%$ and spectral efficiency by $2 \% \sim 36 \%$. Compared with previous BWAFMDA configurations, the new scheme is able to improve energy efficiency by $6 \% \sim 68 \%$ and spectral efficiency by $15 \% \sim 55 \%$. The exact gains depend on network configurations and transmit power levels.

The last article "Flexible UL-DL Switching Point in TDD Cellular Local Area Wireless Networks" investigates uplink-downlink switching techniques for time division duplex wireless networks. An advantage of time division duplex wireless networks over frequency division duplex is that uplink-downlink switching points may be flexibly adapted to asymmetric traffic loads. This enables more efficient spectrum use, but may lead to harmful cross-link interference between cells. 
As a result, the net gain (or loss) from flexible uplinkdownlink switching depends on the traffic characteristics and network scenarios. The authors (Pekka Jänis, Cássio B. Ribeiro and Visa Koivunen) analyze the uplinkdownlink switching problem in femtocell networks where high fluctuations in short-term traffic loads occur. The authors show through theoretical analysis and system-level simulations that in such a scenario significant gains in effective user throughput and packet delays may be achieved under low traffic loads. The gains become smaller at high traffic loads where packets accumulate in queues and there are both uplink and downlink traffics to transmit with high probabilities. When the uplink and downlink transmit powers are not balanced and uplink-downlink switching point adaptation is applied, the link direction with lower transmit power may suffer from degraded performance. The authors show that an interference-aware scheduler provides a better performance and lowers the packet delays further.

The guest editors are thankful to our reviewers for their efforts in reviewing the received manuscripts. We also thank the Edit-in-Chief, Imrich Chlamtac, Ronila Gamatero, Leonora Panday and Sara Fruner for their supportive guidance during the entire process.

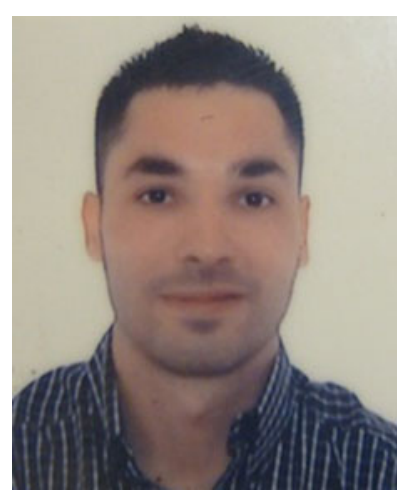

Dr. David Lopez-Perez is a Research Engineer at the Autonomous Networks and System Research Department at Bell Labs, Alcatel-Lucent, Dublin, Ireland, working on Wireless Networking and Small Cells. Prior to this, David received his Bachelor (BSc) and Master (MSc) degrees in Telecommunication from Miguel Hernandez University, Spain, in Sept. 2003 and Sept. 2006, respectively, and his Doctor in Philosophy $(\mathrm{PhD})$ title from University of Bedfordshire, UK, in April. 2011. From Aug. 2010 until Dec. 2011, David was Research Associate, carrying post-doctoral studies, at the Centre for Telecommunications Research (CTR) at King's College London (KCL), London UK. Moreover, from Feb. 2006 and for a year, he was with Cork Institute of Technology, Ireland, and from Feb. 2005 and for a year, he was with VODAFONE Spain, Spain, working at the Radio Frequency Department in the area of network planning and optimization. David has been invited researcher at DOCOMO USA labs, Palo Alto, CA in 2011, and CITI INSA, Lyon, France in 2009. In May 2007, he was awarded with a PhD Marie-Curie fellowship at the Centre for Wireless Network Design (CWiND) at University of Bedfordshire, UK. With 30 years of age, he has published more than 50 book chapters, journal and conference papers in recognized venues, and has been awarded as Exemplary Reviewer for IEEE Communications Letters. He is or has been guest editor of ACM Springer Mobile Networks and Applications
(MONE) Journal and EURASIP Journal of Computer Networks and Communications (JCNC), and editor and/or author of several cellular HetNet related books, i.e. "Heterogeneous Cellular Networks: Theory, Simulation and Deployment" Cambridge University Press, 2012, "Femtocells - Technologies and Deployment", Wiley 2010, and "Femtocell Networks: Deployment, PHY Techniques, and Resource Management", Cambridge University Press, 2012. Moreover, he is or has also been co-chair of several HetNet related workshops, e.g., the 1st IEEE WCNC Workshop on Broadband Femtocells: Paving the way to HetNets, the 2nd IEEE 2011 GLOBECOM Workshop on Femtocell Networks (FEMnet).

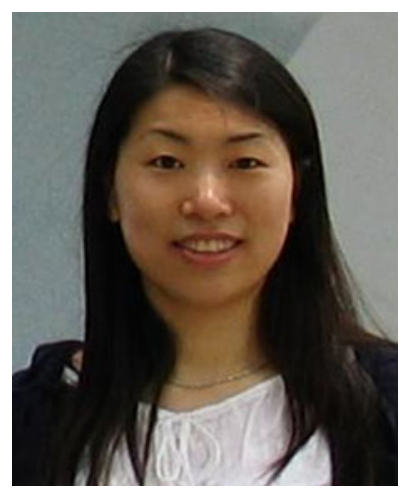

Dr. Xiaoli Chu is a lecturer in the Department of Electronic and Electrical Engineering at the University of Sheffield, UK. She received the B.Eng. degree with first class honours in Electronic and Information Engineering from Xi'an Jiao Tong University, China, in 2001, and the $\mathrm{Ph} . \mathrm{D}$. degree in Electrical and Electronic Engineering from the Hong Kong University of Science and Technology in 2005. From September 2005 to April 2012, she was with the Centre for Telecommunications Research at King's College London, UK. Her current research interests include heterogeneous networks, cooperative communications, cognitive communications, and green radios. She has published more than 40 journal and conference papers and book chapters. She is a guest editor of the Special Issue on Cooperative Femtocell Networks for ACM/Springer Journal of Mobile Networks \& Applications. She has also been Technical Program Committee Co-Chair of several workshops for IEEE and other international conferences. She received the UK EPSRC Cooperative Awards in Science and Engineering for New Academics in 2008, the UK EPSRC First Grant in 2009, and the RCUK UK-China Science Bridges Fellowship in 2011. She is Chair of the Graduates of the Last Decade Affinity Group and Secretary of the Computer Chapter within the IEEE United Kingdom and Republic of Ireland Section.

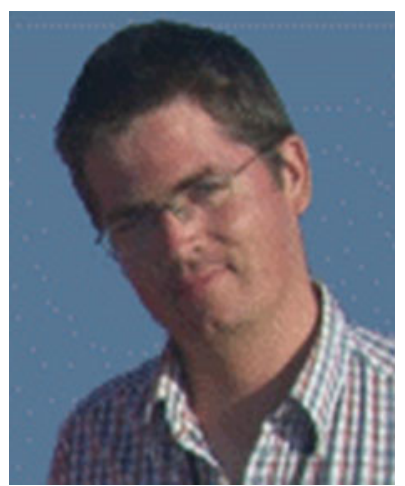

Dr. Guillaume de la Roche is a Wireless System Engineer at Mindspeed Technologies, a leading provider of system on chips (SoCs) for small cells. Prior to that he was with Infineon (2001-2002, Germany), Sygmum (2003-2004, France), CITI Laboratory (2004 2007, France) and Centre for Wireless Network Design (2007-2011, United Kingdom). He has been a part time lecturer at Lyon 1 University since 2005 . He was also a visiting researcher at DOCOMOLabs (2010, USA) and Axis Teknologies (2011, USA). He holds a Dipl-Ing from CPE Lyon, and a MSc and PhD from INSA Lyon. He is a coauthor of approx 60 publications including 3 books. He has been involved in the organizing 
committee for femtocell related events, including IEEE International Workshop on Broadband Femtocell Technologies, IEEE Workshop on Cooperative Heterogeneous Networks and International Workshop on Femtocells. He is involved in the small cell forum (formerly known as femtoforum) activities. He was also a Panelist for the session onfemtocells at the IEEE International Conference on Communications (ICC 2009). He was the principal investigator of European FP7 project "CWNetPlan" on combined indoor/outdoor wireless network planning. $\mathrm{He}$ is a Expert Evaluator for the european commission (FP7). He is on the editorial board of the Transactions on Emerging Telecommunications Technologies (Wiley).

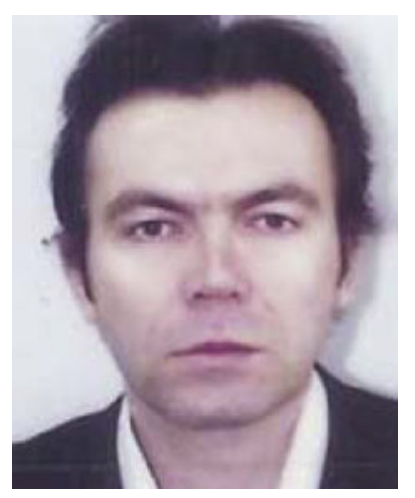

Prof. Athanasios V. Vasilakos is currently Professor at the Dept. of Computer and Telecommunications Engineering, University of Western Macedonia, Greece and visiting Professor at the Graduate Programme of the Dept. of Electrical and Computer Engineering, National Technical University of Athens (NTUA). He has authored or co-authored over 200 technical papers in major international journals and conferences. He is author/coauthor of five books, 20 book chapters in the areas of communications. He served as general chair, TPC chair and symposium chair for many international conferences. He served or is serving as an Editor or/and Guest Editor for many technical journals, i.e., IEEE TNSM, IEEE TSMC-partB, IEEE TITB, JSAC. He is founding Editor-in-chief of the journals: International Journal of Adaptive and Autonomous Communications Systems (IJAACS, http://www.inderscience.com/ijaacs), International Journal of Arts and Technology (IJART, http://www.inderscience.com/ijart). He is chairman of the European Alliance for Innovation.

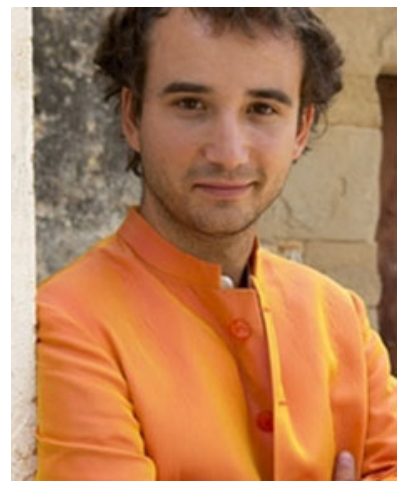

Dr Mischa Dohler is now leading the Intelligent Energy [IQe] group at CTTC in Barcelona, with focus on Smart Grids and Green Radios. $\mathrm{He}$ is working on machine-tomachine, wireless sensor, femto, cooperative, cognitive and docitive networks. In the framework of the Mobile VCE, he has pioneered research on distributed cooperative space-time encoded communication systems, dating back to December 1999. He has published more than 150 technical journal and conference papers at a citation h-index of 29 and citation g-index of 61, holds a dozen patents, authored, co-edited and contributed to 19 books, has given more than 30 international short-courses, and participated in ETSI, IETF and other standardisation activities. He has been TPC member and co-chair of various conferences, such as technical chair of IEEE PIMRC 2008 held in Cannes, France. He is Editor-in-Chief of ETT and is/has been holding various editorial positions for numerous IEEE and non-IEEE journals. He is Senior Member of the IEEE and Distinguished Lecturer of IEEE ComSoc. He had press coverage by BBC, Wall Street Journal, among others. He is fluent in 6 languages. From June 2005 to February 2008, he has been Senior Research Expert in the R\&D division of France Telecom, France. From September 2003 to June 2005, he has been lecturer at King's College London, UK. At that time, he has also been London Technology Network Business Fellow receiving Anglo-Saxon business training, as well as Student Representative of the IEEE UKRI Section and member of the Student Activity Committee of IEEE Region 8 (Europe, Africa, Middle-East and Russia). He obtained his PhD in Telecommunications from King's College London, UK, in 2003, his Diploma in Electrical Engineering from Dresden University of Technology, Germany, in 2000, and his MSc degree in Telecommunications from King's College London, UK, in 1999. Prior to Telecommunications, he studied Physics in Moscow. He has won various competitions in Mathematics and Physics, and participated in the 3rd round of the International Physics Olympics for Germany. 